\title{
TERAHERTZ COHERENT SYNCHROTRON RADIATION FROM FEMTOSECOND LASER MODULATION OF THE ELECTRON BEAM AT THE ADVANCED LIGHT SOURCE*
}

\author{
J.M. Byrd, Z. Hao, M. C. Martin, D.S. Robin, F. Sannibale, R.W. Schoenlein, A.A. Zholents, M.S. \\ Zolotorev, LBNL Berkeley, CA 94720, USA
}

\begin{abstract}
At the Advanced Light Source (ALS), the 'femtoslicing' beamline is in operation since 1999 for the production of $\mathrm{x}$-ray synchrotron radiation pulses with femtosecond duration. The mechanism used for generating the short $\mathrm{x}$-ray pulses induces at the same time temporary structures in the electron bunch longitudinal distribution with very short characteristic length. Such structures emit intense coherent synchrotron radiation (CSR) in the terahertz frequency range. These CSR pulses were first observed at the ALS, and the measurement of their intensity is now routinely used as a diagnostics for the tune-up of the femtoslicing x-ray experiments. At the same time, these CSR pulses synchronous with the modulating laser, represent a potential source of terahertz radiation with very interesting features. Several measurements have been performed for their characterization and in this paper we present an updated description of the experimental results and of their interpretation. In particular, we include more data on the interesting interaction, previously observed at the ALS, between the slicing and the microbunching instability (MBI), where under particular circumstances, the slicing seems to trigger the onset of the instability.
\end{abstract}

\section{INTRODUCTION}

In the 'femtoslicing' experiment at the ALS [1], the copropagation inside a wiggler of a femtosecond laser pulse with the electron beam generates a modulation of the energy in a very short slice of the beam. When such a beam propagates in a dispersive region, the energy modulated particles are transversely displaced and by a proper collimation of the synchrotron radiation (SR), femtosecond pulses of $\mathrm{x}$-rays are produced [2]. At the same time, due to the finite momentum compaction of the ring (longitudinal dispersion), the modulation in energy induces a density variation in the longitudinal distribution when the beam propagates along the ring. The characteristic length of such longitudinal structures starts from femtosecond duration right after the undulator, quickly increases up to the ps order and practically disappears in about one ring turn. It has been predicted that such structures should radiate coherent synchrotron radiation (CSR) in the terahertz frequency range [3] and our group at the ALS has provided the first experimental evidence of that [4]. The intensity

\footnotetext{
*Work Supported by the Director, Office of Science, of the U.S Department of Energy under Contract No. DE-AC03-76SF00098
}

signal of these CSR pulses, measured by a bolometer, are now routinely used as diagnostic for the tune-up of the femtoslicing experiment. In fact, the best transverse and longitudinal overlap between the laser pulse and the electron beam maximizes the $\mathrm{THz}$ CSR intensity.

In the first section of this paper, we present time and frequency domain measurements of the CSR induced by the slicing and their comparison with predictions.

In the second section, we show more experimental data on a particular phenomenon first observed at the ALS [4] when the femtoslicing is performed with a particular lattice and above the threshold for the CSR induced microbunching instability (MBI) [5-7]. In such a situation, the slicing seems to triggers the onset of the instability, the CSR bursts associated with the MBI become synchronous with the laser and their average intensity grows exponentially with the current per the bunch.

\section{CSR FROM FEMTOSLICING}

The relevant parameters of the ALS and of the femtoslicing experiment during the described experiments are shown in Table 1.

Table 1: ALS and Femtoslicing Experiment Parameters

\begin{tabular}{|l|c|c|}
\hline Parameter & BL 5.3.1 & BL 1.4 \\
\hline Modul.-observ. point distance [m] & 8.4 & 149.5 \\
\hline Energy [GeV] & \multicolumn{2}{|c|}{1.5} \\
\hline Current per bunch [mA] & $1-10$ \\
\hline Ring length [m] & 196.7 \\
\hline Dipole bending radius [m] & 4.957 \\
\hline Momentum compaction & 0.00137 \\
\hline Relative energy spread & 0.001 \\
\hline Relative energy modulation & 0.006 \\
\hline Laser pulse duration FWHM [fs] & 70 \\
\hline Laser repetition rate [pps] & 1000 \\
\hline
\end{tabular}

For the measurements, we used the SR emitted by dipole magnets at two different ALS beamlines. The first one, BL 5.3.1, is the closest available SR port downstream the straight section where the modulating wiggler is located $(\sim 1 / 24$ of the ring length). Unfortunately, this port has a small vertical acceptance $(\sim 2.5 \mathrm{mrad})$ and presents a poor transmission for the large divergence $\mathrm{THz}$ SR. The second beamline, BL1.4, is about $3 / 4$ of the ring length downstream the wiggler and it is the ALS beamline dedicated to IR experiments. BL1.4 presents a larger acceptance $(10 \mathrm{mrad})$ and 
operates under vacuum preventing absorption of $\mathrm{THz}$ SR from water vapor.

Using Table 1 parameters, we calculated the slicing induced modulation of the electron bunch longitudinal distribution at the two different beamlines. The results are shown in Figure 1.
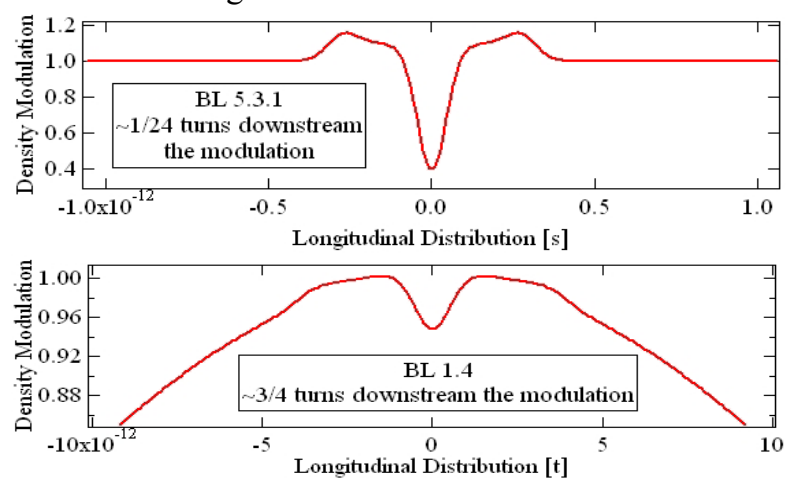

Figure 1: Slicing induced modulation of the electron beam longitudinal distribution calculated at the two ALS beamlines.

The structures shown in the figure are short enough to radiate $\mathrm{CSR}$ in the $\mathrm{THz}$ frequency range [3] and the associated spectrum of the radiation evolves from higher to lower frequencies along the ring following the progressive lengthening of the bunch modulation.
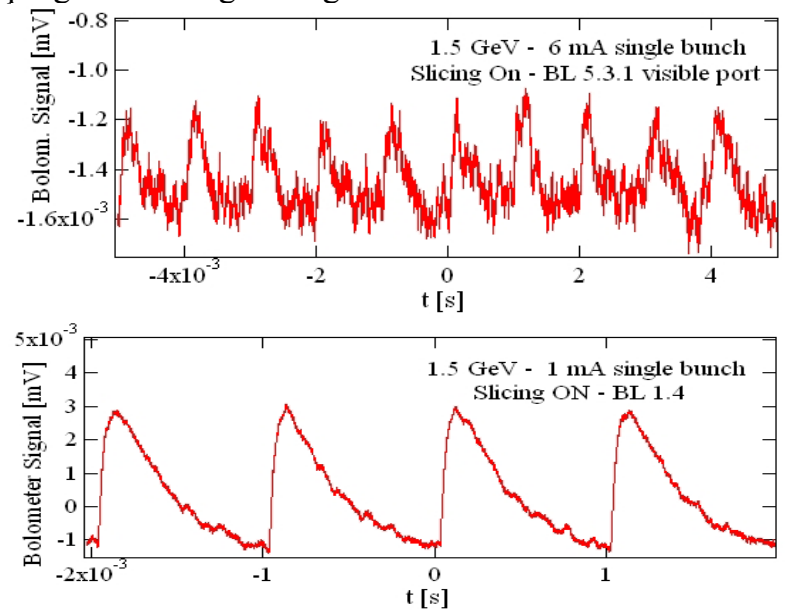

Figure 2: Bolometer signal at a scope during slicing at the two beamlines.

By using a bolometer, we measured the CSR power at both beamlines and we found a clear quadratic dependence versus the current per bunch confirming the coherent nature of the radiation. Figure 2 shows an example of the bolometer signal at a scope. The $1 \mathrm{kHz}$ repetition rate of the laser is clearly visible while the shape of the pulse is completely dominated by the time response of the detector. More details on our experimental setup can be found elsewhere [4].

We also performed spectra measurements using a spectrometer at both the beamlines. Figure 3 shows an example of the measurements. The shape of the spectrum measured at BL 5.3.1 is due to the combination of several effects. The water vapor in the non in vacuum channel absorbed some frequencies of the radiation creating the small structures visible in the spectrum.
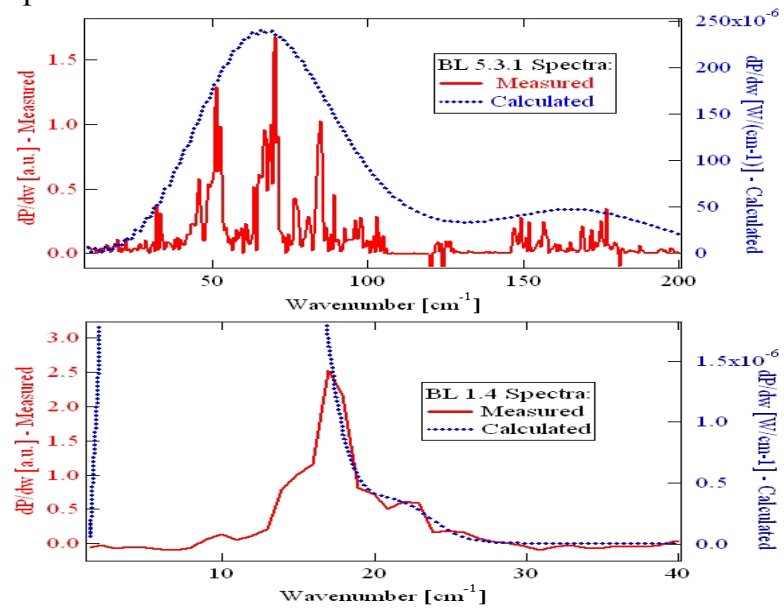

Figure 3: Calculated and measured spectra of the CSR induced during slicing at the two beamlines

For the larger structures (as for example the 'holes' in the spectrum between 30 and $40 \mathrm{~cm}^{-1}, 55$ and $65 \mathrm{~cm}^{-1}$, 75 and $85 \mathrm{~cm}^{-1}$ ), we think they are due to the interference of the radiation inside the long and narrow gap that the SR must go through when leaving the vacuum chamber (waveguide-like effect). Simulations with a simplified geometry using SRW [8] qualitatively confirmed such a hypothesis. Additionally, the small acceptance of the beamline severely cuts the intensity of the CSR making the measurement noisy (see Fig.2).

The quality of the spectra taken at BL1.4 was much better, but because of the instrumentation bandwidth limitation and of the vacuum chamber cut-off frequency it was possible to measure only the higher frequency part of the spectrum.

Nonetheless, the comparison in Fig. 3 between the measured spectra and the calculated ones shows a reasonable agreement for both the beamline cases. In calculating the spectra in Fig. 3 we used the values in Table 1 adjusting some of them within less that $10 \%$ in order to have a better matching with the data. This is consistent with the magnitude of the experimental errors.

\section{SEEDED CSR BURSTING}

During our $\mathrm{THz}$ experiments with slicing, in order to modify the spectrum of the induced CSR, we used an ALS lattice with momentum compaction of 0.0027 , about twice the one of the regular operation lattice. With this particular lattice and above the current threshold for the MBI, we started to see a strong anomalous increase of the signal from our bolometer.

The MBI is a single bunch instability induced by the $\mathrm{SR}$ wakes [5-7]. It generates microstructures in the longitudinal distribution of the bunch that can last for several turns and that are short enough to emit strong bursts of CSR in the terahertz frequency range. The 
time structure of such bursts is generally random and their amplitude shows large fluctuations.
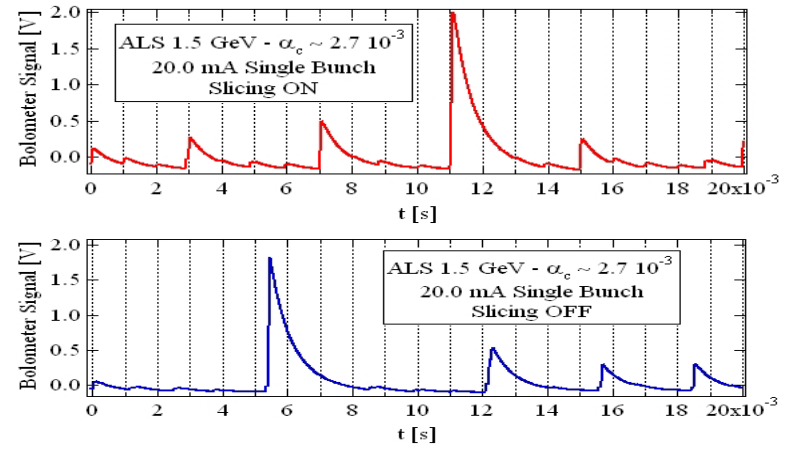

Figure 4: Bolometer signal with the double momentum compaction lattice and above the MBI current threshold. Top: slicing on; bottom: slicing off.

With the new lattice and above the MBI threshold, we observed that most of the CSR bursts became synchronous with the $1 \mathrm{kHz}$ repetition rate of the slicing laser, see Fig. 4, and that the power started to grow exponentially with the current per bunch [4].

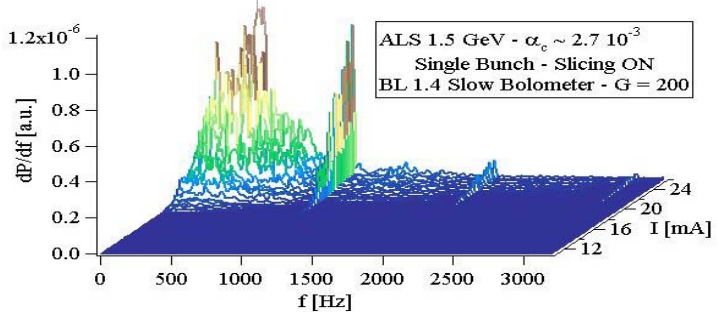

Figure 5: Fourier transform of the bolometer signal for different currents per bunch.

Figure 5, shows the Fourier transform of the bolometer signals for different currents per bunch. Two things are evident from the figure, the onset of the instability at about $16 \mathrm{~mA}$ and the $1 \mathrm{kHz}$ line associated with the laser repetition frequency. Figure 6 shows the amplitude of this $\mathrm{kHz}$ line versus current. The data are nicely fitted by an exponential curve with imposed saturation.

We also measured the seeded bursts at BL 1.4 with a faster detector $(800 \mathrm{kHz}$ bandwidth). Figure 7 shows the signal at the scope triggered with the $1 \mathrm{kHz}$ laser trigger. In the top of the figure the scope is in sample acquisition mode while in the bottom is in the average one. The first pulse in the tracks is due to the CSR emitted by the density modulation right after the slicing and lasts for a single turn. About $30 \mu$ s later, a large number of pulses due to CSR bursts are shown. Some uncorrelated bursts are also visible.

A possible interpretation of the observed data is the following. The MBI is normally triggered by spontaneous density fluctuations (noise) in the beam longitudinal distribution. The MBI threshold depends on the current per bunch and on the wavelength of the SR under observation, the shorter the wavelength the higher the threshold [5, 7]. A density fluctuation becomes unstable when its characteristic length is comparable to the wavelength under observation $(\sim 0.1$ $1 \mathrm{~mm}$ for $\mathrm{THz}$ ) and if the number of particles per bunch is larger than the MBI threshold for that wavelength. When the beam is 'sliced', the fluctuation generated by the modulating laser has a very short characteristic length and thus a very high MBI threshold and cannot trigger the onset of the instability.

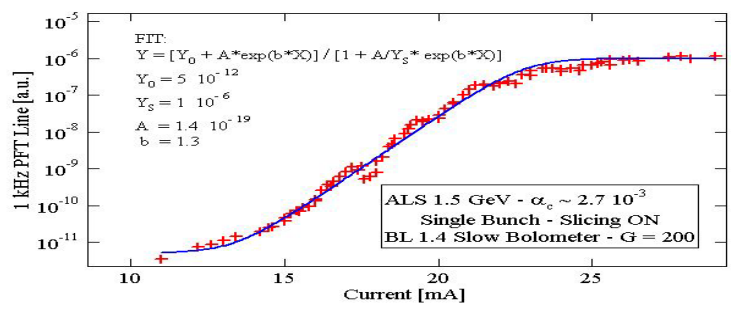

Figure 6: CSR intensity as a function of the current per bunch with the 'double' momentum compaction lattice.

The density modulation quickly disappears in one turn but after half a synchrotron period $(\sim 30 \mu$ s for this ALS lattice) it is back again but this time with a larger characteristic length due to lattice nonlinearities and to $\mathrm{SR}$ emission. If the current is now above the MBI threshold for this 'new' length the instability will be triggered. Future effort and measurements will be dedicated to the verification of this or other hypotheses.

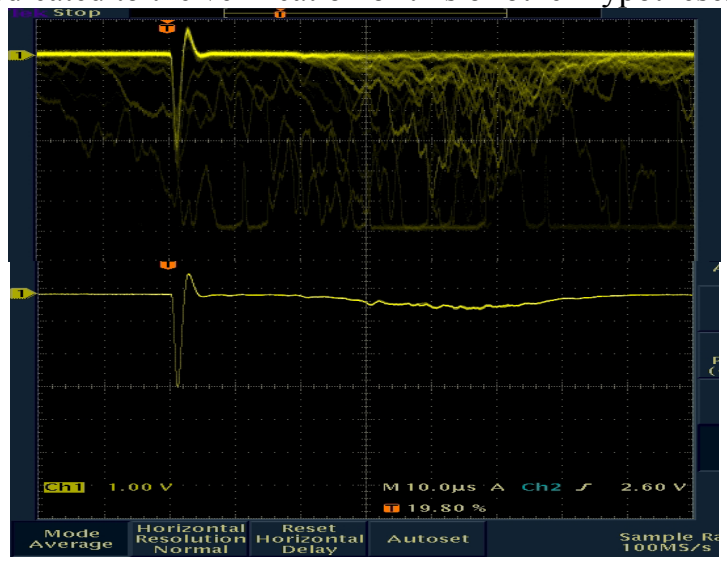

Figure 7: Scope tracks of the seeded burst signal at the fast detector. Scales: $10 \mu \mathrm{s} / \mathrm{div}$ and $1 \mathrm{~V} / \mathrm{div}$

\section{REFERENCES}

[1] R.W.Schoenlein, et al., Science, Mar 24, (2000) 2237.

[2] A.Zholents, M.Zolotorev, Phys. Rev. Lett. 76, 912, (1996).

[3] R.W.Schoenlein, et al., Appl.Phys.B 71, 1-10, 2000.

[4] J. M. Byrd et al., Proceedings of the European Part. Acc. Conf. EPAC04, Lucerne, Switzerland, July 12-16, 2004, pag. 2448.

[5] G. Stupakov and S. Heifets, Phys. Rev. ST 5, 054402 (2002).

[6] J.M.Byrd et al., Phys. Rev. Lett. 89, 224801 (2002).

[7] M. Venturini and R. Warnock, Phys. Rev. Lett. 89, 224802 (2002).

[8] O.Chubar, P.Ellaume, www.esrf.fr/Accelerators/Gr oups/InsertionDevices/Software/Radiations/SRW. 
